\title{
An Energetic Cognitive Radio Network Communication Principles with Attack Identification Strategies using Game Theory
}

Thanga Revathi S ( $\boldsymbol{\nabla}$ thangars@srmist.edu.in )

SRM Institute of Science and Technology

\section{Gayathri A}

Saveetha Institute of Medical and Technical Sciences: Saveetha University

\section{Kalaivani J}

SRM Institute of Science and Technology

\section{Manickam M}

SRM Institute of Science and Technology

\section{Kalaiselvi K}

SRM Institute of Science and Technology

Deepa Thilak K

SRM Institute of Science and Technology

\section{Research Article}

Keywords: Cognitive Radio Network, Primary User Impersonation, Energetic Cognitive Radio Network Game Planner, Game Theory, Time and Energy Sharing

Posted Date: May 27th, 2021

DOl: https://doi.org/10.21203/rs.3.rs-556672/v1

License: (c) (1) This work is licensed under a Creative Commons Attribution 4.0 International License. Read Full License 


\section{Abstract}

In a wireless communication industry Cognitive Radio Network is the most important concern to deal with long range communications in efficient manner with futuristic remote sensing and channel allocation policies. This kind of communication principles need to tackle the attacks in wide manner. Generally, the attacks come from the intruder end with several variations but the most common attacks are network sniffing, route trapping and so on, but in the case of Cognitive Radio Network the major threat is called Primary User Impersonation (PUI). This kind of PUI attacks acts like a regular Primary User (PU) and raise a signals accordingly to make confuse the Secondary Users to come out from the communication line as well as the entire communication spectrum utilized by the Secondary User gets affected. Due to these kinds of affections the legitimate Secondary Users suffer and left from the respective channels utilized for communication. This type of attacks highly creates an impact over the Cognitive Radio Network and causes the failure over spectrum accessing. The flexible accessibility by some voluntary users maintains a constant selection of actions to determine their appropriate target, so they can differentiate between accomplishing their objectives and being compliant with their goals. The critical role is protected against obstruction and moreover mandated by vitality criteria. At the aim to develop energy saving and power gathering cooperative spectrum, factors that are central to the success of the project must be suggested in the literature as two major protocols, such as: Time Sharing (TS) and Energy Sharing (ES). An energy utilization constraint restriction is imposed on the power cells (battery) to make sure they do not charge and discharge at around the parallel period Primary User Impersonation detection in an efficient manner, in which it uses recent advancements in cooperative spectrum sensing to devise an algorithm called Energetic Cognitive Radio Network Game Planner (ECRNGP). A Game Theory logic provides an intelligent attack identification abilities to the proposed approach to identify the threat signals immediately and notify that to the respective transmitter unit to make appropriate preventive measures for that as well as this proposed approach efficiently identifies the Primary User Impersonation and provides a sufficient protection to the CRN environment as well as Secondary Users to make a perfect communication between entities without any attack oriented constraints.

\section{Introduction}

The present wireless communication and networking infrastructure limits how much it can evolve, making it inadequate lack of broad responsiveness, low on flexibility, a network made up of these elements (comprising nodes, protocols, and policies) is unable to change The hierarchical configuration of network environment reduces the transmission of network information, thereby leaving the different layers ignorant of the status of the network. For each participant in the network, only a small answer is availableand while anything goes wrong then do the alterations to the network do something creative [1]. Measuring data released by the Federal-Communications-Commission indicates that approved frequency ranges are largely progressively used. In order to get the most out of the scarce spectrum they are investigating cognitive radios, which are able to adjust their operational characteristics to match the channel conditions and it is an intelligent wireless system that utilizes any existing operation, channel- 
constraints, encoded data, or transmissions of Primary Users In keeping with the administratively and financially flexible options, Secondary Users plan to submit overlay, or make use, on-the-the-fly clients [2] [3]. This segment contains additional information on the various cognitive radio paradigms and the following sections discuss regarding the boundaries of each schema in detail.

\section{A. Cognitive Radio Network Environment}

If the Cognitive Radio Network becomes more capable, it becomes increasingly important to implement many, different network modes such as peer-to-peer networking, multi-service networks and carrier assisted cognitive networking paradigms. It is useful to look at three large network paradigms, such as underlaying network environment, overlaying-network environment and interweaving-network environment. In other words, there is a higher limit to the amount of interference they can cause to each primary user and an equivalent or lower amount of output each primary user is supposed to experience [7]. In an overlaying-network environment, Secondary Users pick up the communication of the primary users and use them to the benefit of both, while learning new signal and processing methods in an improvement of their own. Secondary Users see when licensed Primary-User signals are not present and can chat opportunistically when necessary [13][14]. For any of the three perfect models, clients, there must be external clients who share data resources with the primary ones and with other clients with different data needs according to their own view of the environment and any other client in the MAC runs the risk tier comes to the forefront here as well as any idea for an alternate Medium-Access-Control meeting which really resides within a single perspective is indeed routine and unoriginal to date [8]. A variety of separate clients, such as a cell or satellite receivers, may send their data to one optional recipient (in the uplink), and one client may send data to a number of different beneficiaries (in the downlink). The underlaying-network environment is illustrated in graphical manner over the following figure, Fig-1; in other words, the secondary transceivers are able to conduct and receive only if the bandwidth presented to the endpoints is sufficient. So rather than evaluating the desired impedance, the device allows a client to choose a higher nominal transmission rate with the anticipation that it will encounter a thin obstacle power phantom underfoot [10]. Any spread is spread to its intended recipients' as well as this distributed range theory is the basis of spread spectrum and Ultra Wide Band (UWB) also known as Ultra Wide Band communications. In parallel, the Secondary User energy level allows the suggested interference to remain well below the total energy yield [15].

\section{B. Game Theoretic Model}

A game theory is the universal gaming logic to improve the efficiency of its appliance, in which it is mostly utilized in communication scenarios to improve the transceiver efficiency level by means of eliminating the threats from the communication scenario. This approach follows the model in which it contains a set of rules and regulations with set of key players in assemble with the environment. This paper utilizes the logic of game theoretic modeling to improve the efficiency of the proposed approach called Energetic Cognitive Radio Network Game Planner (ECRNGP). This game theoretic model provides the solution to the problems such as resource allocation and route analysis. In parallel, the game 
theoretic approach identifies the resource allocation issues raised over the wireless network environment and provides a proper solution to those issues. In Cognitive Radio Network environment the communication between transmitter and receiver entities are handled based on the licensed channel availabilities and the correlation of Primary Users and the Secondary Users. This kind of communication scenario creates a bond between PU and the SU to take care of one and another to provide a success ratio high and an efficient wireless network communication between transceivers. However the communication boundaries need to be threat free and efficient, in which the attack avoidance abilities need to be improvised in such CRN environments by means of some powerful network maintenance logic such as proposed ECRNGP, in which it applies the game theoretic logic with wireless communication network handling strategies, so that the communication metrics are clearly monitored by the game theoretic routing principles and take an opportunistic routing between entities to provide a flaw free communication model. The following figure, Fig-2 illustrates the game theoretic model cycle establishments with the proposed approach of ECRNGP, in which it creates a defender module to eliminate the risk metrics through the network system training principles and make protective mechanisms to the available cognitive radio network users (both PUs and SUs).

The major contributions of this paper are as follows:

i. To improve the attack preventive measures to the Cognitive Radio Network by means of applying some anti-threat policies to the network environment.

ii. A new methodology is designed to provide a flaw-free communication model between transceivers with anti-threatening features.

iii. To improve the channel allocation efficiency by avoiding the network overhead cased by attackers as well as to improve the spectrum sensing principles by using Secondary User management schemes such as Game Theoretic Modeling.

iv. A new approach called Energetic Cognitive Radio Network Game Planner is designed with high level of network management efficiency in order to provide a high-level communication support without any network interference.

The rest of this paper describe regarding Related Study over Sect. 2, further section of Sect. 3 illustrates the proposed system methodologies in detail with proper algorithm flow and the Sect. 4 illustrates the Result and Discussion portion of the paper and the final section, Sect. 5 illustrates the concept of Conclusion and Future Scope of the proposed paper. These all will be explained in detail over the further section summaries.

\section{li. Related Study}

Prativa-Rai et al., 2020 [4], proposed a paper related to performance level improvements over Cognitive Radio Network using Game Theoretic Model with respect to utility associated methodologies. In this 
paper [4], the authors illustrated such as Cognitive-Radio based Wireless Communication Networks are really common now and carefully planning channel in a network can be a complex undertaking. The game theoretic models can also covers those situations and a lot of research is unusable. However, when deciding on functions for the allocation scheme, it is crucial to consider how to allocate channels according to how much utility they can produce. Utility methodologies that can be implemented in GTbased resource scheduling strategies are studied in this paper [4] and it has been explained in great depth how to provide different channels to various nodes using a game theoretic approach based network resource allocation method. Creative solutions were put forward for three related potential uses for utility simulations and a planning projection of this implementation [4] is described in clear manner.

Dongjian Liu et al., 2020 [5], proposed a paper related to resource and spectrum allocation strategies over satellite interfacing with the ground, it becomes clear that the satellite communication can take off, because the more this system [5] utilize regarding that and it becomes more efficient. For the last few years, satellite and broadband connectivity has caused stagnation in the growth of the satellite grid. With the introduction of intelligent radio, bandwidth will be used more effectively and the interference problems among satellites and broadband networks will be addressed. The use of several satellites by Secondary-Users and Primary-Users has been presented in this research [5], in which it is helpful to recognize the channel condition of Secondary Users. Within this circumstance, Secondary User plays a game theoretic methodically decides on the network latency and then allocates the bandwidth Based on the findings, there are consistent solutions for both the gaming phase and the connectivity of PU, it was shown that the wireless spectrum can be equally and appropriately used [5].

Jili-Wang et al., 2020 [6], proposed a paper related to a game theory enabled spectrum sharing model on Cognitive Radio Network environment the architecture proposed in this paper [6] establishes a novel spectrum sharing mechanism for Cognitive Radio Satellite networks. Because CRN users tend to be based outside of or on the fixed satellite system, the cognitive radio network users apply various techniques around within or outside the range of the satellite. The first attempt to look at multi-channel connectivity and spectrum efficiency is handled in this paper [6] under distributed architectures initially. A multi-channel game and energy automation have thus been devised that virtualizes through cognitive user. Since the findings of the different studies confirm these two game-theoretic models must have at least one Nash-Equilibrium Logic. Hopefully soon, a multi channel game access and game power optimization model is suggested as well as Consistency and Supremacy of the proposed algorithm [6] was verified by the resultant of simulation outcomes.

Shilian-Zheng' et al., 2020, [11] proposed a paper related to Cognitive Radio Networks based Spectrum analysis strategies using deep learning principles. In this paper [11], the authors presented such as spectrum sensing is a key cognitive radios technology and it represents a problem of classification as well as proposed a deep learning classification sensing technique. The signal power obtained is normalized to overcome the effects of noise uncertainty and these strategies are training the model with as many signals and acoustic data as possible so that the trained network structure can respond to untrained new signals. It also use transmission learning method to promote legitimate signal 
performance and the extensive studies are carried out to assess the method's performance. The implementation results indicate that the proposed process is better than two conventional methods of spectrum sensing, that is the superior and lowest self-value-based method and the signal enabled frequency domain method. The research performance of the proposed undertrained signal categories also shows that our approach can be adapted to detect these levels of importance. In addition, legitimate signal detection results show that transmission learning can further increase classification accuracy. Finally, colored noise experiments showed that the proposed method has improved colored noise detection performance, while the classic methods show a major degeneration in performance that confirms the uniqueness of the proposed methodology [11].

Arnab-Barua, et al., 2020 [12] proposed a paper related to channel data analyzing with respect to Cognitive Radio Network intelligence and its associated methodologies. In this paper [12], the authors presented such as cognitive processing and networking have been used in many communication sectors in recent decades and the current improvements in deep learning process and BigData estimations case tremendous potential for cognitive intelligence $(\mathrm{Cl})$ analysis for many applications such as monitoring and recognition of human activities over communication technology. The creation of intelligent healthcare systems is related to cognitive abilities and communications systems. Health assisted surveillance strategies are becoming extremely interesting topics in which the researchers focus today on the monitoring of postoperative hospitalized patients. In this paper we argue that profound learning and wireless communication techniques bring cognitive intelligence to the system of health monitoring. We present a profound learning neural network model (CNN) that classifies image data and a convenient and multifunctional Software Defined Network environment, in which it detects movement of ankle fracture patients. The main idea of this research is to capture wireless location information in the context of the nervous system and to classify it and use Convolutional Neural Network methodology to examine distinct motions. A Universal-Peripheral-Software environment is used to capture and classify wireless location information. Alex-Net and ZF-Net are both the popular Convolutional Neural Network architecture and it is used to classify trapped images from quantitative values in random manner. The classification developed between diagnosis and characterization resulting on the knee movements shows that Convolutional Neural Network produces significant improvement where detection rate is around 98.98 percent.

\section{lii. Proposed System Methodologies}

In this paper, a new Cognitive Radio Network communication model is designed called as Energetic Cognitive Radio Network Game Planner (ECRNGP), in which it provides efficient communication strategies over the network environment with attack free scenario by using game theoretic modeling. This approach provides a robust detection scheme against Primary User Impersonation (PUI) attack raised on Cognitive Radio Network environment. This kind of attack identifies the signal pattern of the Primary User and creates the same type of signal patterns over the network environment to force the legitimate Secondary Users to provide the required spectrum for communication, in which it degrades the overall network performance as well as the Secondary Users suffer a lot due to this attack. 
The suggested solution called ECRNGP is unusual in that, in which it includes non-predictable workloads and it has no limitations regarding the quantity of Primary Users and the Secondary Users. The operation pattern of a signal is measured by spectrum sensing and the proposed algorithm then recreates the observed signal frequency pattern by using a construct to predict potential activity. The suggested ECRNGP scheme uses statistical analysis to differentiate between Primary User signals and attacks. The main goal is that while an attacker can influence the signal, the system identifies that and can't influence the composite. Attackers are able to relay Primary User, although this differs from other Primary User identification mechanism, in which it predicts the Primary User interference by means of game theoretic modeling. Usually, the attacker attempts to reduce the bandwidth on the Cognitive Radio Network, e.g., by making it unavailable at specific times of increasing the ON time intervals or decreasing the OFF time intervals. But the proposed approach identifies the periodic intervals by means of applying the different

Service Access architectural schema to variate the deviations. To put it in another way, if an attacker only cheats on the curve, for example by adjusting their baseline level, the proposed scheme of ECRNGP contend that that this contradicts the denial-of-service goal of the attacker. This is due to the fact that a Cognitive Radio Network chooses the low occupied spectrum operations platform as their focus of interest. The major objective of the proposed approach is to reconstruct the Service Access nature by means of adjusting the communication vectors B1, B2, B3,..., Bn. The allocated resources are tied up with the estimations of communication vectors. These functionalities are defined by using the following equation.

\section{$\mathbf{Y} \leftarrow \mathbf{E}+\sum_{i=1}^{n} B_{i} \mathbf{W}$}

Where the weight factor Wi is deviated from 1 to $n$, specifically $1<=\mathrm{i}<=n$. The base vectors for communication spectrum ranges are reconstructed with respect to $\mathrm{Y}$ and the training model carefully reorganizes that based on service level architectures. The following algorithm illustrates the flow of the proposed model called Energetic Cognitive Radio Network Game Planner in detail with process flow details.

\section{Algorithm}

\section{Energetic Cognitive Radio Network Game Planner}

1. if Cognitive Radio Network setup is initialized first time as new, then analyze the Spectrum ranges.

2. Inspect the spectrum level by analyzing the service level architectures, in which it is represented as $X 1, x 2, X 3, \ldots, X n$.

3. Initiate the For Loop from 1 to the spectrum range $n$, in which it is acquired from spectrum maximum range $(X n)$.

4. $f \circ r(i=0 ; i<=n ; i++)\{\}$

5. Estimate the mean and average value with respect to service level CRN architectures. 
6. Analyze the training level mean and average value with respect to the testing samples identified in Step-5.

7. Analyze the tolerance limit of the spectrum variance till ON state of the network.

8. Estimate the least tolerance rate of the network OFF state.

9. Manipulate the average mean and variance level with respect to the estimated vector based on Eq. (1).

10. Estimate the maximum and least tolerance rate of the network processing and service level initiations.

11. Indicate the attack level and spectrum range to the respective transceivers with proper specifications.

12. Exit from the loop.

\section{Iv. Results And Discussions}

This paper designed a new game theoretic plan called Energetic Cognitive Radio Network Game Planner, in which it identifies the Primary User Impersonation (PUI) attack efficiently by means of its prediction strategies as well as the signal vector alterations. The proposed approach is developed by using a powerful network simulation tool called Network Simulator and the parameter ranges are customized based on dynamic aspects, so that the user can dynamically set the parameters according to their convenience and outcome requirements. The following table, Table-1 illustrates the parameter specifications of the proposed approach.

\section{Table-1 Input Parameters}

\begin{tabular}{|ll|}
\hline Input Parameters & Value \\
\hline Predefined Decision Threshold to decide whether PU is absent or not( $\mathrm{T})$ & $20-50 \%$ \\
\hline CooperativeDetectionProbability & 40 to $60 \%$ \\
\hline Transmission Packet Size & $100-1000 \mathrm{bits}$ \\
\hline Packet Transmission Speed & $500-1000 \mathrm{kbps}$ \\
\hline Network Receiving Ability & $200-750 \mathrm{kbps}$ \\
\hline PUs Maximum Transmission Power & $100-1000 \mathrm{kbps}$ \\
\hline SUs Maximum Transmission Power & $50-500 \mathrm{kbps}$ \\
\hline Average Delay & $10-50 \mathrm{~ms}$ \\
\hline Average Node Minimum Energy Consumption Level & $10-50 \mathrm{~J}$ \\
\hline
\end{tabular}

The figure, Fig-3 illustrates the training model creation principles, in which this training phase execution allows the proposed model to accurately find the failures immediately as well as the affected nodes from 
the CRN without any further delay. This training model leads a way to successful communication between entities presented into the Cognitive Radio Network.

The following figure, Fig-4 illustrates the overall Cognitive Radio Network formation with proper positioning of Primary and Secondary Users with respect to proposed approach ECRNGP strategy as well as this approach concentrates more on network routing configurations, in which it will be defined by means of Dynamic Source Routing protocol and the distance metrics are clearly estimated with respect to dynamic link layer principles.

The figure, Fig-5 illustrates the attack identification in clear manner with respect to the battery draining condition, in which the attacker intensity is to drain the battery level by wasting the spectrum ratio in Secondary User end. This process repeatedly happening means, the overall energy level and the spectrum ratio will goes down as well as it leads to the overall performance degradation of the entire Cognitive Radio Network, so that the proposed approach identify the attack and recover the affection by means of applying the idle nodes battery power to the drained nodes and reactivate that affected node from the affection level to the normal condition. This process does not leave any network node to get affected at any case, so that the network performance level will be maintained as it is without any interference.

(a)

(b)

(c)

Figure 5 (a) Attack Identification (b) Node Recoveryand (c) Recovered Node Perception

The figure, Fig- 6 illustrates the Cognitive Radio network environment channel spectrum sensing ratio in clear manner with practical outcome, in which the x-axis indicates the frequency level of the channel and the $y$-axis indicates the power/energy level ratio in graphical manner.

The figure, Fig-7 illustrates the Cognitive Radio network environmentpacket loss scenario of the proposed approach, in which it cross-validate the proposed approach ECRNGP packet loss scenario with training model and without training classical model, in which the trained model provides the highest impact on outcome and the proof is clearly demonstrated in graphical manner.

The figure, Fig-8 illustrates the Cognitive Radio network environment average channel sensing level of the proposed approach, in which it cross-validate the proposed approach ECRNGP channel sensing level with training model and without training classical model, in which the trained model provides an improved channel sensing ratio as compared with the classical model and the proof is clearly demonstrated in graphical manner.

The figure, Fig-9 illustrates the Cognitive Radio network environment energy consumption ratio analysis as well as the proposed approach trained model is cross-validated with the existing non-training model. 
The resulting structure of proposed model is far better than the classical model and the resulting proof is provided in graphical manner over the following figure.

The figure, Fig-10 illustrates the Cognitive Radio network environment Battery Consumption level analysis as well as the proposed approach trained model is cross-validated with the existing non-training model. The battery level estimations and the associated consumption ratio estimations are the major metrics of CRN. The outcome ratio is far better than the classical model and the resulting proof is provided in graphical manner over the following figure.

\section{Conclusion And Future Scope}

The Primary User Impersonation (PUI) detection system termed Energetic Cognitive Radio Network Game Planner (ECRNGP), which acquires the Signal Activity Pattern of the Primary User signal transmitters. The proposed approach uses a Game Theoretic model to reconstruct the scenario to organize determined signal activities and the associated node strength related details without any interference. Completely different from current solutions on the Primary User Impersonation (PUI) detection, ECRNGP doesn't want a priori information of Primary Users as well as has no limitation on the kind of applicable Primary Users. The performance analysis indicates that ECRNGP is strong and effective to sight each straight and smart Primary User Impersonation attackers, despite the fact that the good attackers might forge the signal activity patterns. The Cognitive Radio Network is subjected to basic signal pattern and the associated game theoretic pattern constraints. This approach had been shown that taking the various harvests, sensing and news characteristics of Secondary Users into thought can yield a stronger performance in terms of realizable throughput, energy consumption and so likelihood of being active. Since the active constraints of associate degree users presented into the $\mathrm{CRN}$ is extremely dependent on network environment, sensing and associated attributes. Given a possible set of Secondary users, deciding the best set of cooperating channel estimation scheme is of the essence to achieve most realizable total outturn.

In future, the work can be further extended by means of adding some deep learning principles to provide a better training experience and it leads to better time savvy process of ECRNGP logic.

\section{Declarations}

We hereby declare that we are the sole author's of this article. To the best of my knowledge this article contains no material previously published by any other person except where due acknowledgement has been made.

\section{References}

1. Tran, T. N., Nguyen, T.-V., Shim, K., \& An, B., "A Game Theory Based Clustering Protocol to Support Multicast Routing in Cognitive Radio Mobile Ad Hoc Networks", IEEE Access, 2020. 
2. Wang, Z., \& Mark, B. L., "Game-Theoretic Framework for Cooperative Relaying in Cognitive Radio Networks", ICC 2020-2020 IEEE International Conference on Communications, 2020.

3. Ning, B., Sun, G., Li, J., Zhang, A., Hao, W., \& Yang, S. "Resource Allocation in Multi-User Cognitive Radio Network With Stackelberg Game", IEEE Access, 2020.

4. Prativa Rai, Mrinal Kanti Ghose and Hiren Kumar Deva Sarma, "An Analysis on the Impact of Utility Functions on the Performance of Game Theory Based Channel Allocation in Cognitive Radio Wireless Sensor Network", IEEE International Conference on Advanced Networks and Telecommunications Systems, 2020.

5. Dongjian Liu, X., Yang, N. X., \& Zhu, S., "A spectrum allocation algorithm for satellite-terrestrial communication based on game theory", International Conference on Information Science, Computer Technology and Transportation, 2020.

6. Jili Wang, D., Guo, \& Zhang, B., "A Novel Spectrum Sharing Scheme for Cognitive Satellite Networks: A game-Theoretic Approach", International Conference on Wireless Communications and Signal Processing, 2020.

7. Christo, M. S., Vasanth, K., \& Varatharajan, R. (2020 Jan). A decision based asymmetrically trimmed modified winsorized median filter for the removal of salt and pepper noise in images and videos. Multimedia Tools and Applications, 79(1), 415-432.

8. Govindaraj, D., \& Logashanmugam, E. (2019). Multimodal verge for scale and pose variant real time face tracking and recognition. Indonesian Journal of Electrical Engineering and Computer Science, 13, 665. 10.11591/ijeecs.v13.i2.pp665-670.

9. Wang, N., Han, S., Lu, Y., Zhu, J., \& Xu, W., "Distributed Energy Efficiency Optimization for Multi-User Cognitive Radio Networks Over MIMO Interference Channels: A Non-Cooperative Game Approach", IEEE Access, 2020.

10. Christo, M. S., \& Meenakshi, S. Enhancing Rumor Riding protocol in P2P network with Cryptographic puzzle through challenge question method. Computers \& Electrical Engineering. 2018 Jan 1;65:122 38.

11. Zheng, S., Chen, S., Qi, P., Zhou, H., \& Yang, X., "Spectrum sensing based on deep learning classification for cognitive radios", China Communications, 2020.

12. Arnab Barua, Z.-Y., Zhang, F., Al-Turjman, \& Yang, X. "Cognitive Intelligence for Monitoring Fractured Post-Surgery Ankle Activity Using Channel Information", IEEE Access, 2020.

13. Moinul Hossain, \& Xie, J. "Hide and Seek: A Markov-Based Defense Strategy Against Off-Sensing Attack in Cognitive Radio Networks", IEEE Transactions on Network Science and Engineering, 2020.

14. Jing Ren, H., Zhang, Z., Du, Youming, S., Hang Hu and Zhu, X. "Weighted-Directed-Hypergraph-Based Spectrum Access for Energy Harvesting Cognitive Radio Sensor Network", IEEE Access, 2020.

\section{Figures}




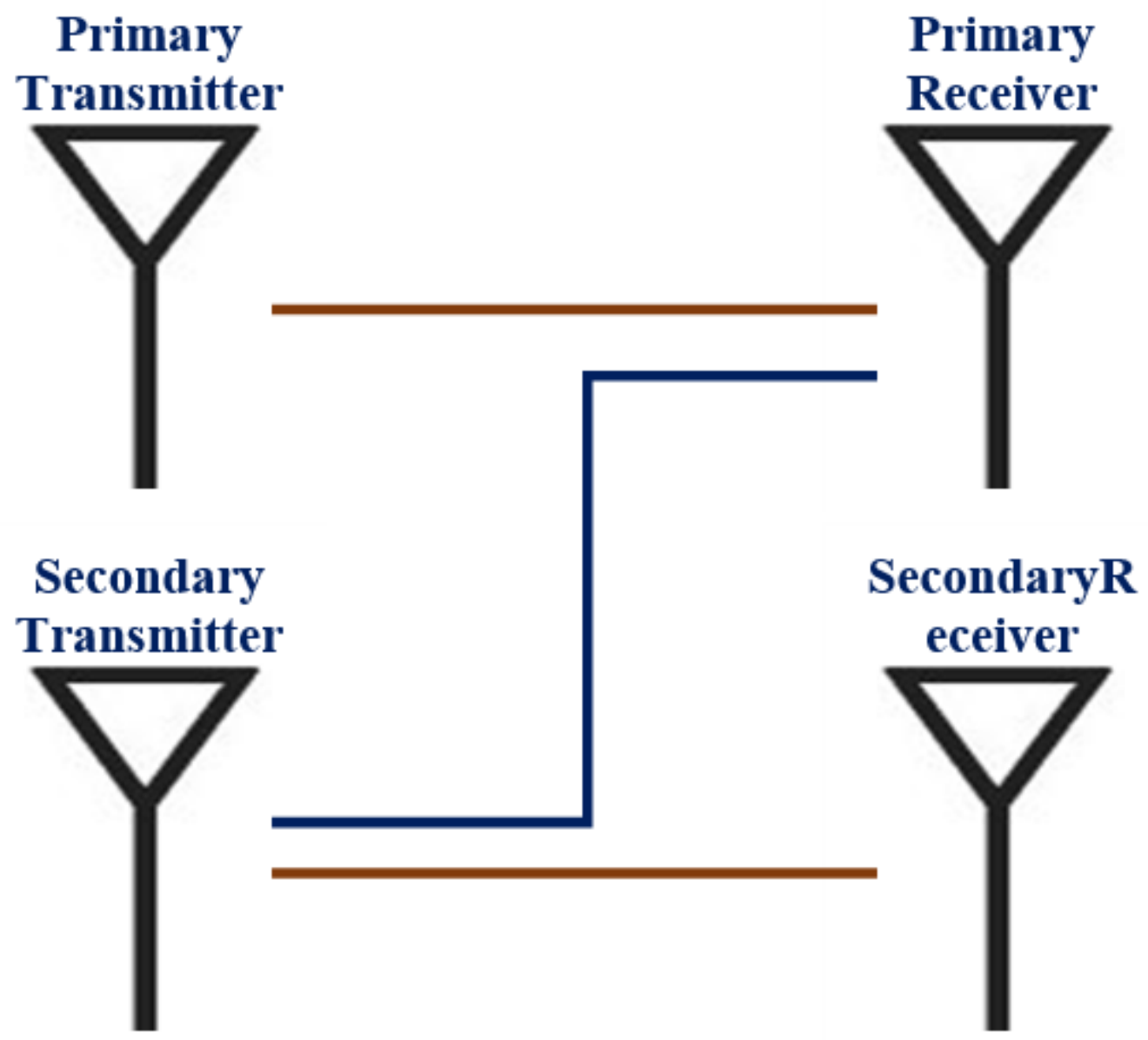

Figure 1

CRN Network Environment with Underlaying Network Architecture 


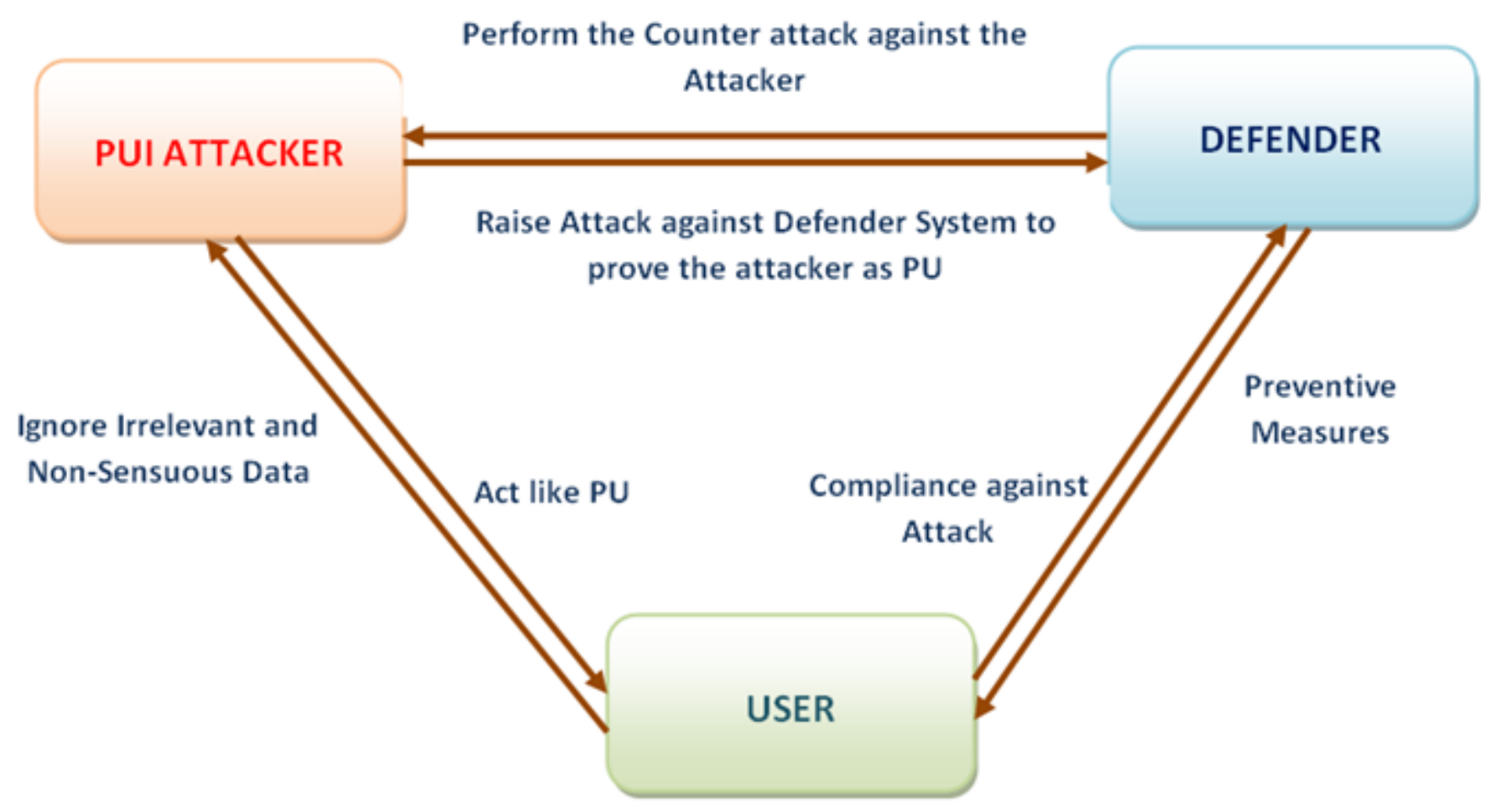

Figure 2

CRN Game Theoretic Architecture

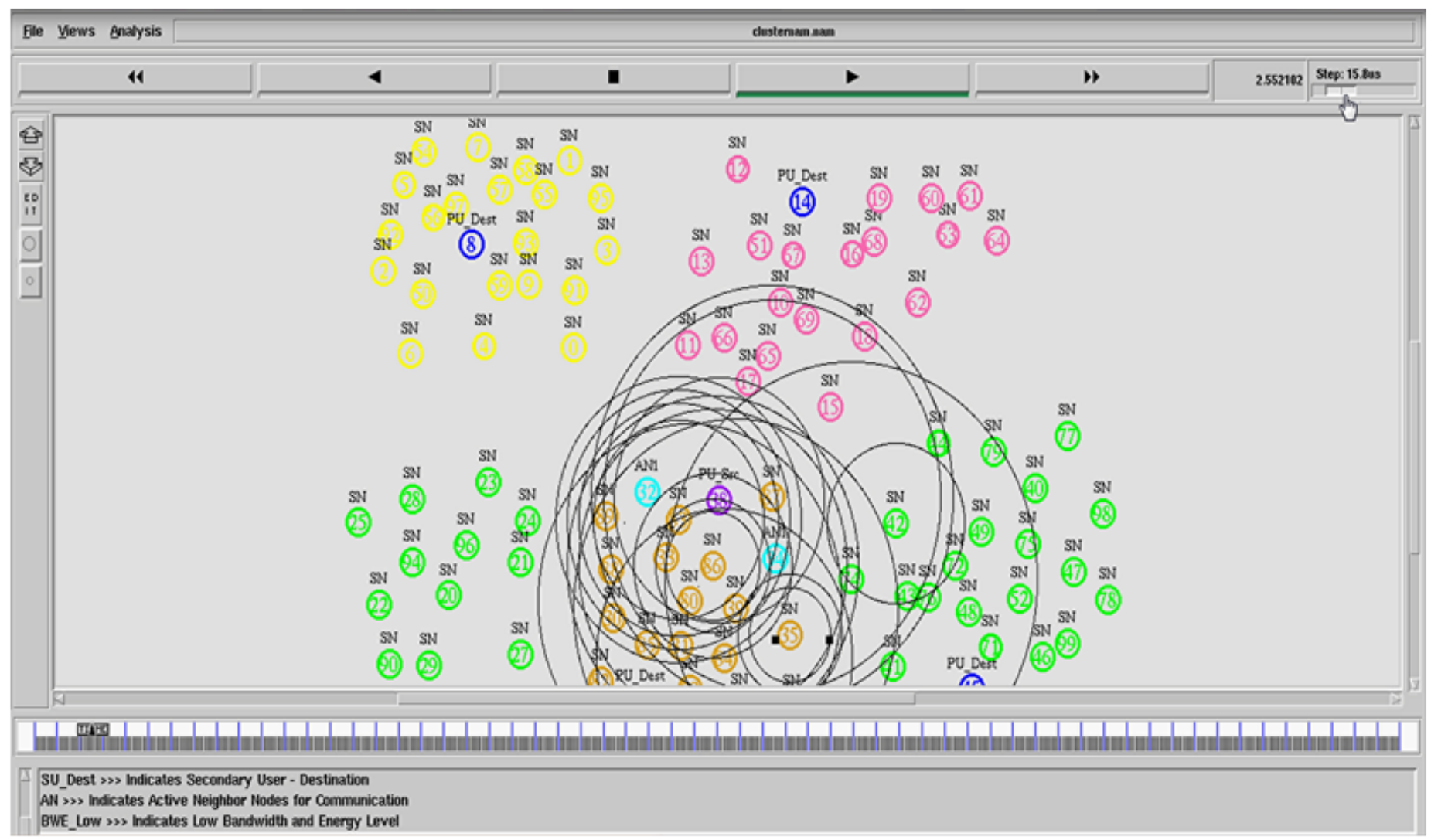


Figure 3

Training Model of CRN

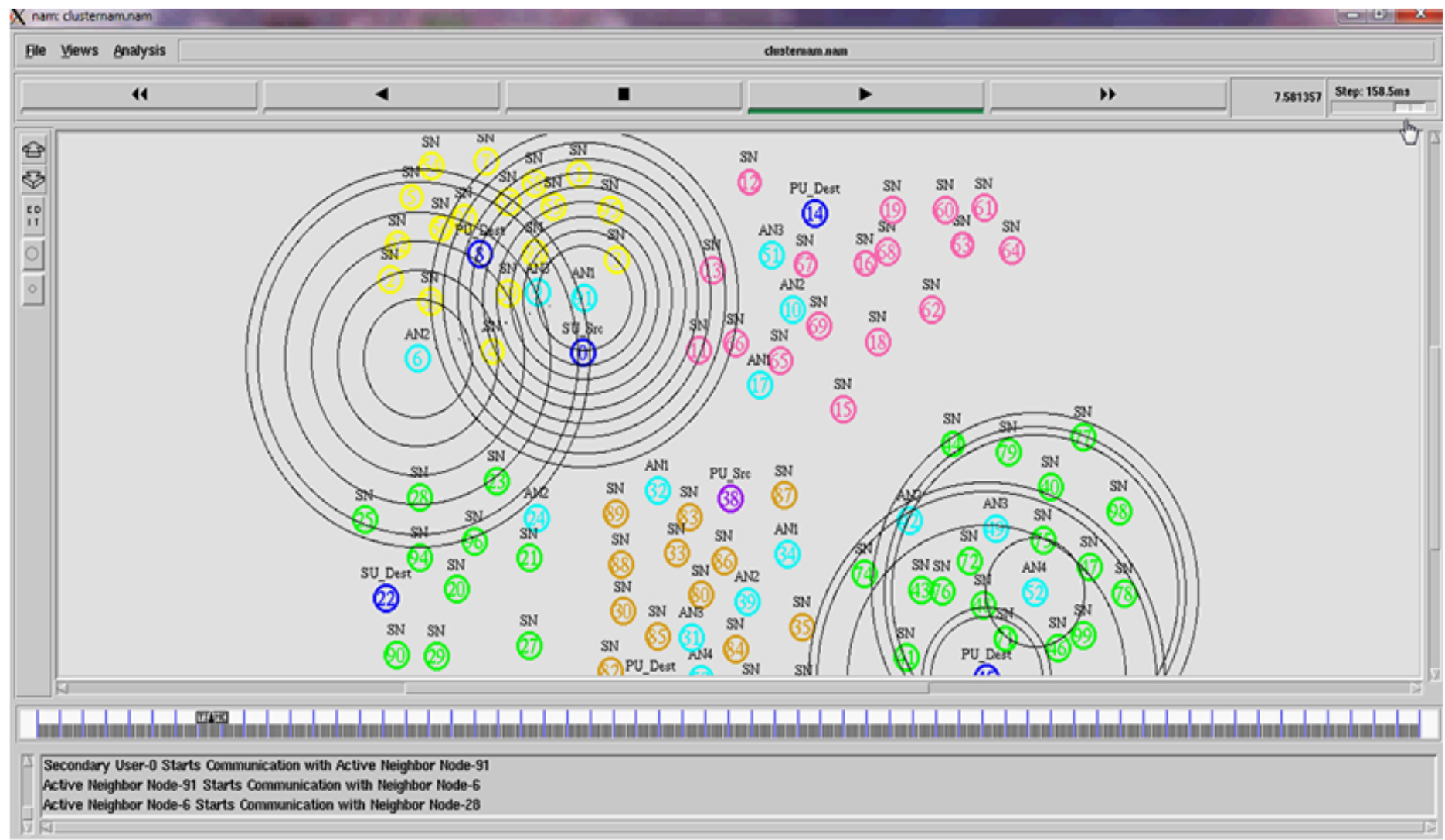

Figure 4

CRN Communication Model 


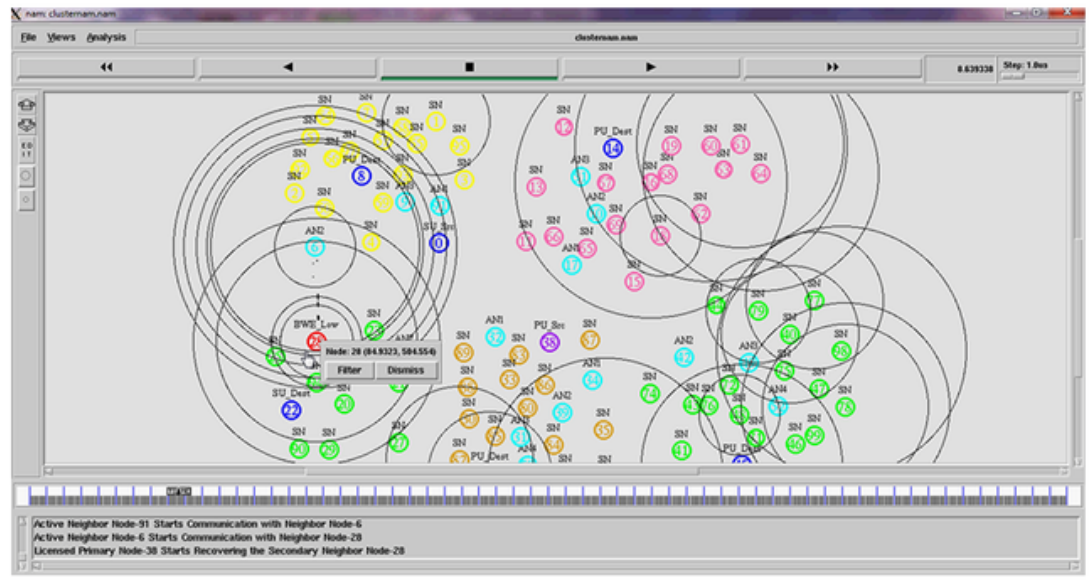

(a)

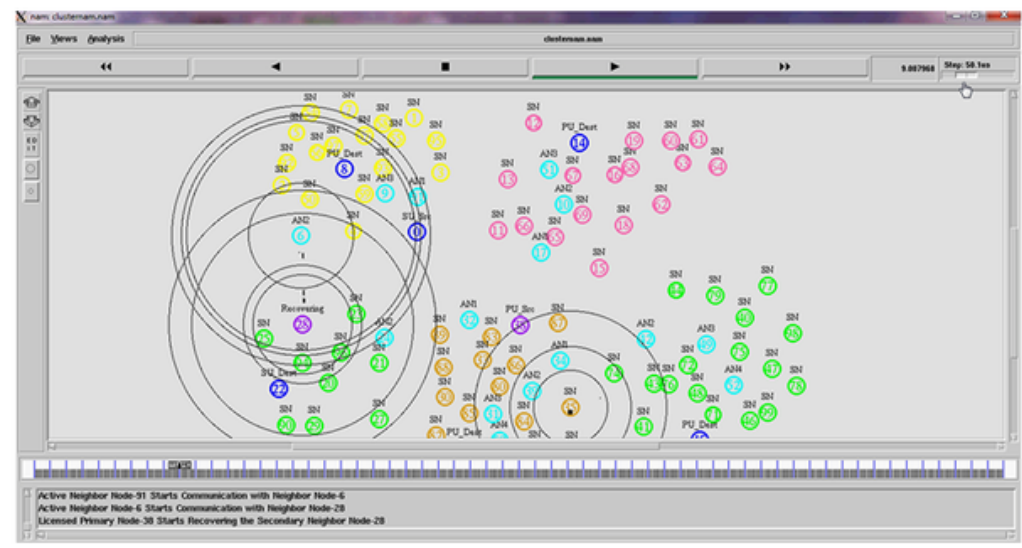

(b)

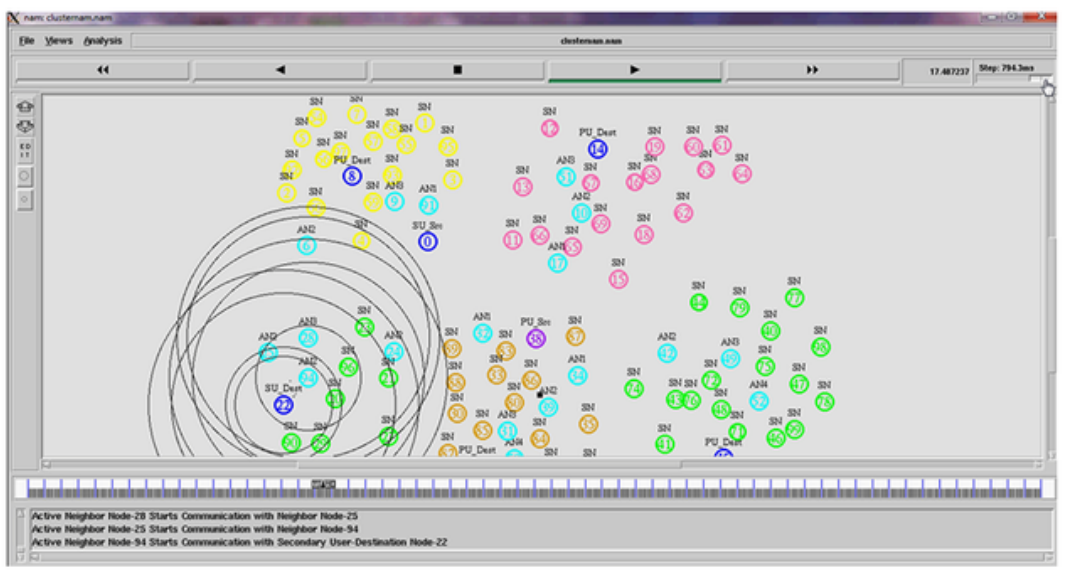

(c)

Figure 5

(a) Attack Identification (b) Node Recoveryand (c) Recovered Node Perception 


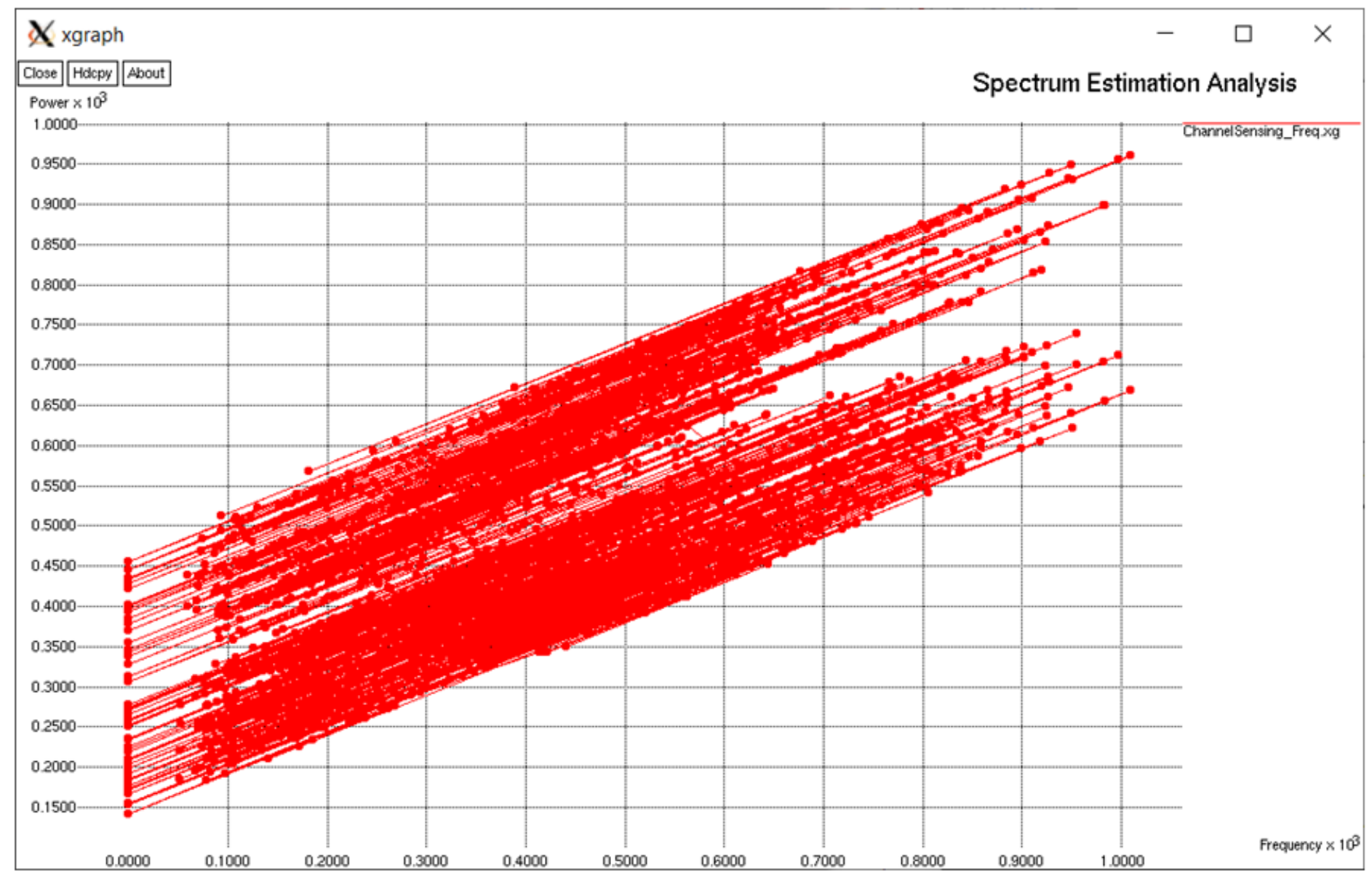

Figure 6

Spectrum Estimation 


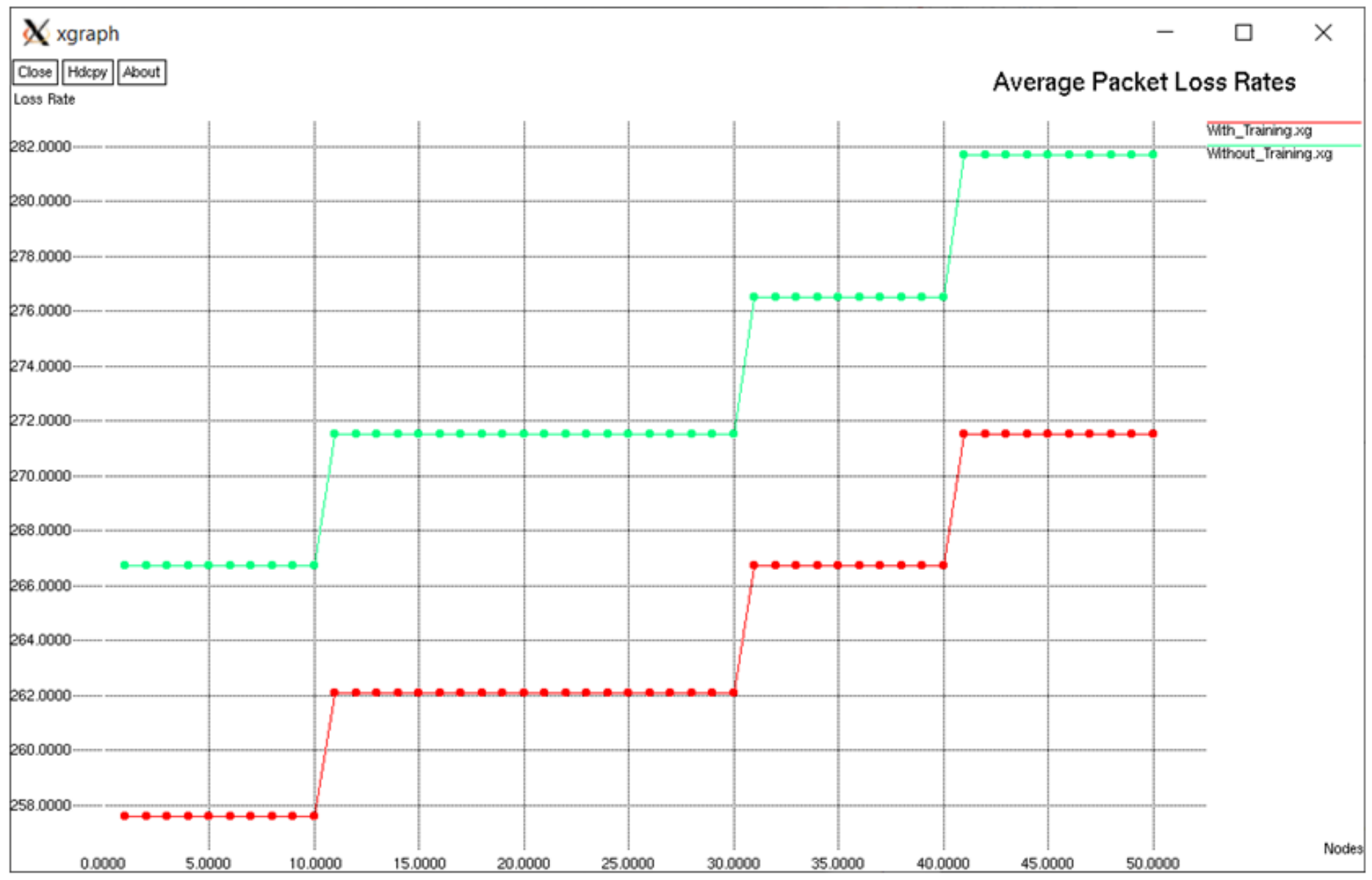

Figure 7

Packet Loss Estimation 


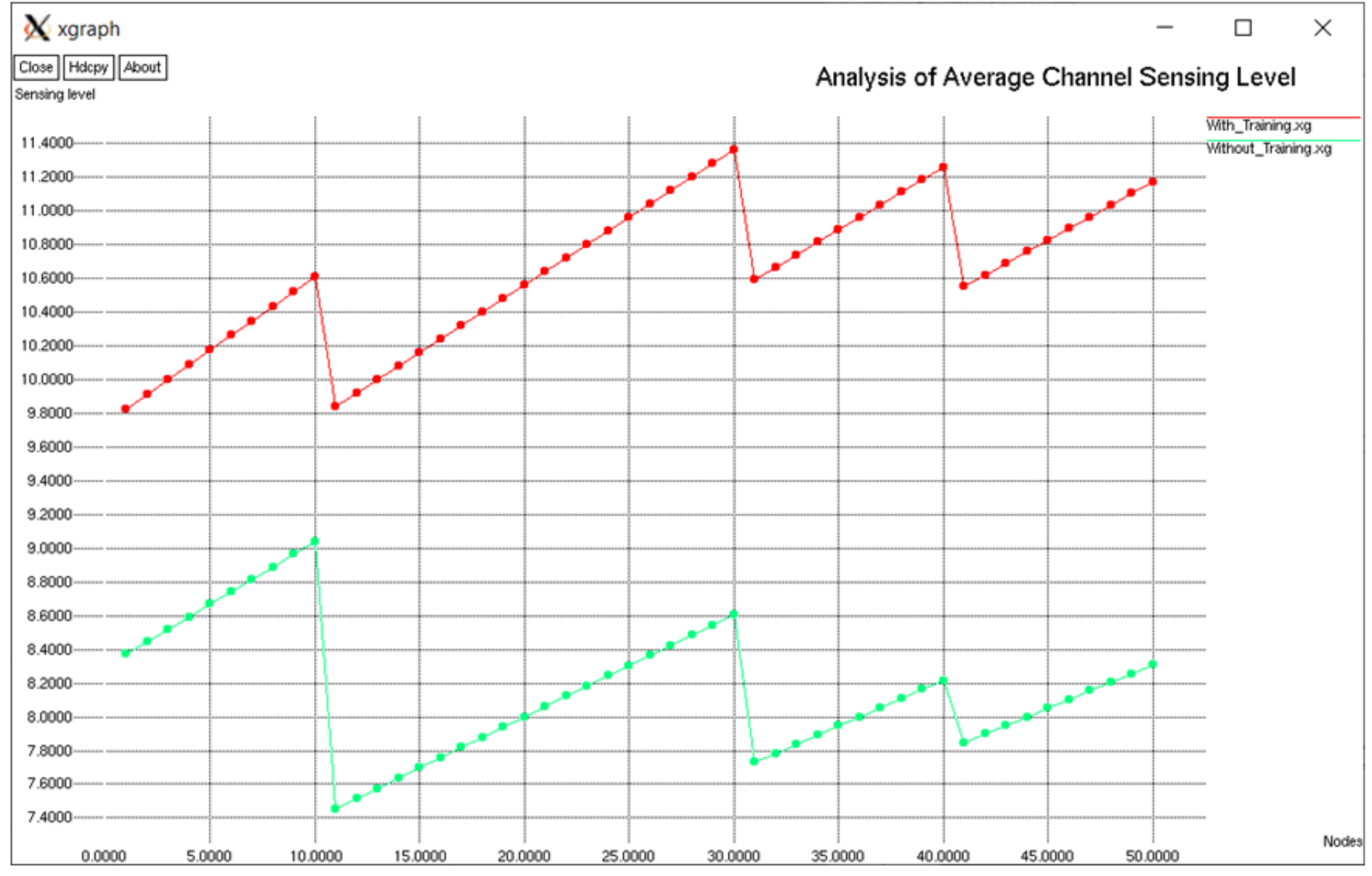

\section{Figure 8}

Channel Sensing Ratio Analysis 


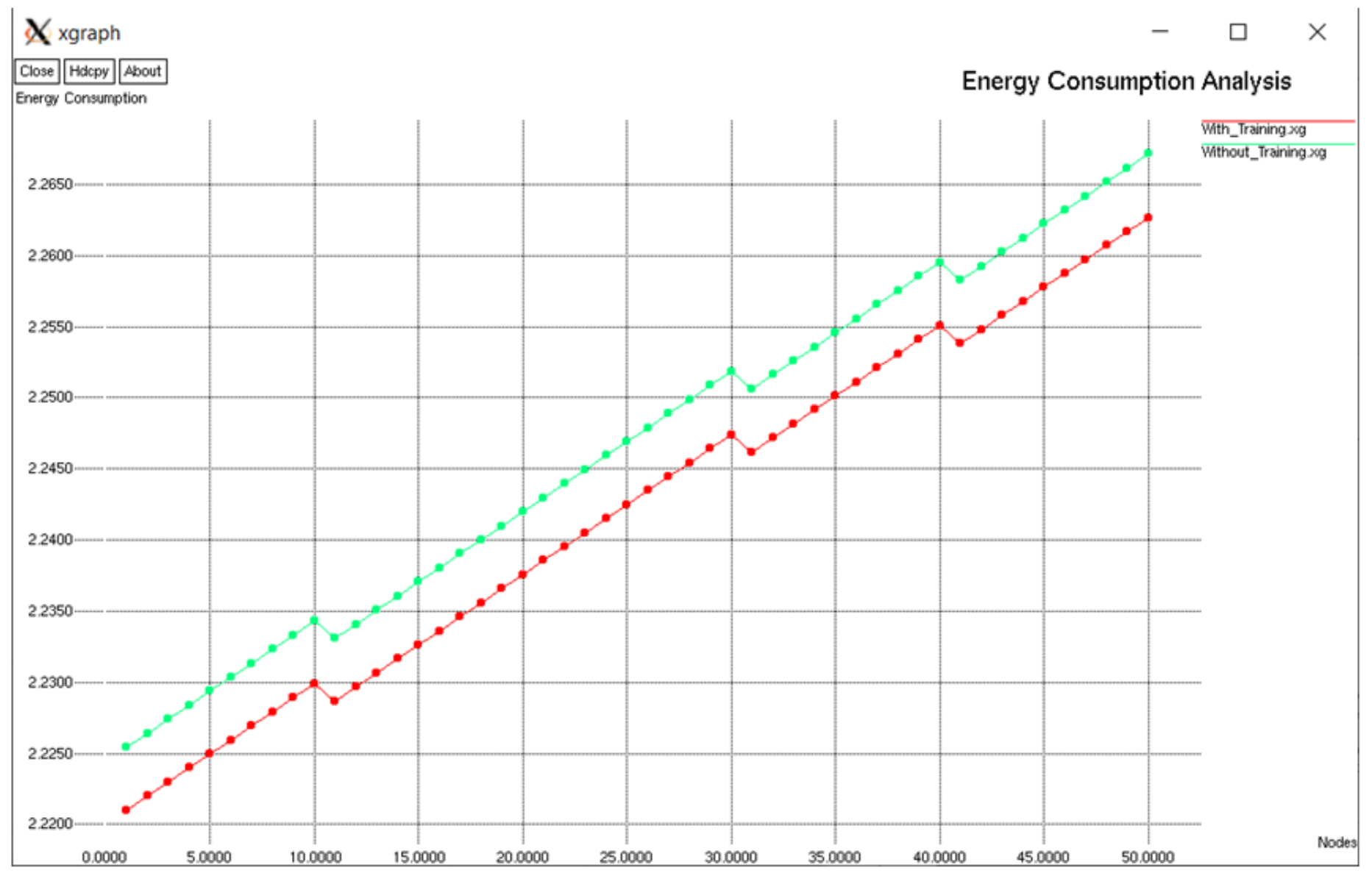

Figure 9

Energy Consumption 


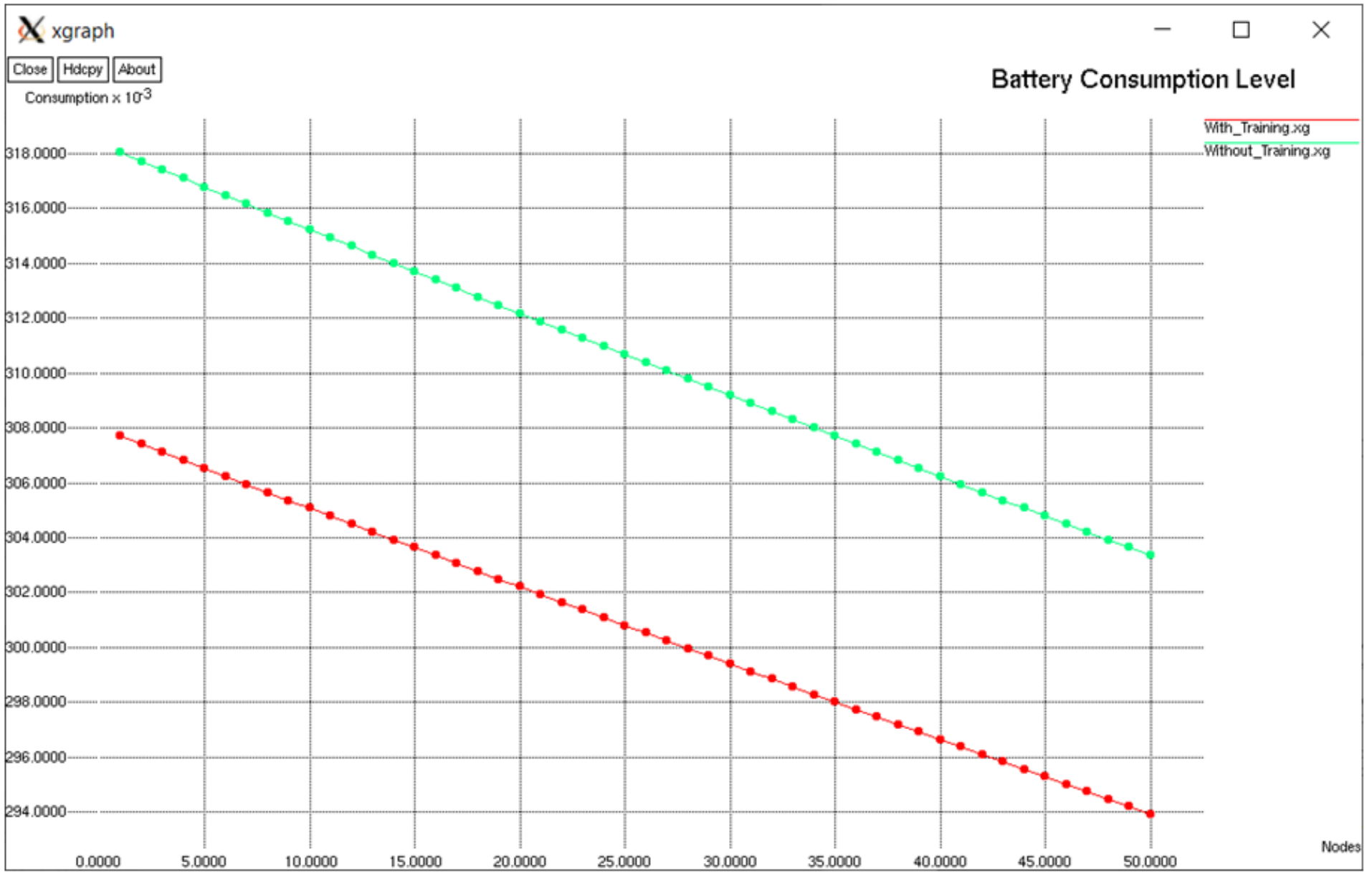

Figure 10

Battery Consumption Analysis 